\title{
Dynamics of Oxidant-antioxidant System in Patients with Multidrug- resistant Tuberculosis Receiving Anti-mycobacterial Therapy
}

\section{Dmytro O Butov ${ }^{1 *}$, Michael M Kuzhko², Irina M Kuznetsova ${ }^{3}$, Olena O Grinishina ${ }^{4}$, Olga A Maksimenko ${ }^{5}$, Tatyana S Butova ${ }^{1}$ and Irina M} Kalmykova ${ }^{6}$

${ }^{1}$ Kharkiv National Medical University, Kharkiv, Ukraine

${ }^{2}$ F.G. Yanovsky National Institute of Phtysiatry \& Pulmonology, National Academy of Medical Sciences, Kiev, Ukraine

${ }^{3}$ Regional TB Hospital № 1, Kharkiv, Ukraine

${ }^{4}$ Regional TB Dispensary № 3, Zmeyev, Ukraine

${ }^{5}$ Regional TB Dispensary № 4, Izyum, Ukraine

${ }^{6}$ Regional TB Dispensary № 1, Kharkiv, Ukraine

\begin{abstract}
The changes in oxidant-antioxidant system were evaluated in serum samples of 140 patients with pulmonary tuberculosis, comprising those with multidrug-resistant TB (MDR-TB) and without MDR-TB and 30 healthy donors. The select parameters of Antioxidant System (AOS) including levels of thiol (-SH) groups, total antioxidant activity, glutathione peroxidase and glutathione reductase as well as metabolites of oxidative degradation of lipids (LPO), i.e., trienoic conjugates and lipofuscin, were measured by spectrophotometry. At treatment initiation AOS and LPO values of TB patients were significantly lower or higher in comparison with healthy donors. After the two months of chemotherapy there was a significant increase of AOS and decrease in LPO values in TB patients. In patients with MDR-TB and relapsed pulmonary TB was noticed more pronounced authentic changes of pro-oxidant-antioxidant system in the blood of the comparison is not MDR TB and newly diagnosed pulmonary tuberculosis as for the standard anti-tuberculosis therapy since two months after treatment.
\end{abstract}

Keywords: Tuberculosis; Multidrug-resistant tuberculosis; Relapsed tuberculosis; Newly diagnosed pulmonary tuberculosis; Antituberculosis therapy; Peroxide oxidation of lipids; Antioxidant system

\section{Introduction}

Tuberculosis (TB) remains the global problem of mankind with 8.8 million new cases of TB diagnosed each year and 1.4 million people dying from the disease $[1,2]$. The emergence of Multi-DrugResistant (MDR) and extensively drug-resistant TB threatens disease control efforts throughout the world [3,4]. About 50 million people are estimated to be infected with resistant strains of Mycobacterium tuberculosis [5].

In the last decade the Ukraine experienced marked increase in the proportion of TB strains resistant to one or more anti-mycobacterial drugs, causing reduced effectiveness of standard chemotherapy $[6,7]$. As a result the frequency of Relapsed or Recurrent Pulmonary Tuberculosis (RPTB) becomes higher [8]. The success of treating relapsed tuberculosis remains low resulting in higher rate of transition to chronic disease and increased mortality $[9,10]$. One of reasons for relapse include disorders in oxidant-antioxidant system, which affect the proper immune surveillance and exaggerates destructive inflammatory process underlying the variety of pathological changes $[11,12]$. Several studies have implicated local and systemic intensification of prooxidant-antioxidant system in various forms of pulmonary tuberculosis [13-15].

Clinical manifestations of $\mathrm{TB}$, especially its progression and outcome, are closely associated with nonspecific metabolic perturbations in patients. TB is characterized by the activation of lipid peroxidation (LPO), reduced activity of Antioxidant System (AOS) and other related changes. These processes can play a significant role in elimination of Mycobacterium tuberculosis, but the imbalance in these processes can also causes damage to the healthy lung tissue and enhancement of inflammatory process [16-19].

One of the basic mechanisms that regulate the stability and permeability of cell membranes are LPO and AOS [16,20]. Normally the LPO, i.e., the formation of reactive oxygen species, is counterbalanced by AOS. Our study was aimed to uncover changes of pro-oxidant-antioxidant system in patients with various forms of RPTB, including those with MDR-TB. We have examined the dynamics of oxidant-antioxidant system in TB patients who were receiving antimycobacterial therapy.

\section{Materials and Methods}

We have followed 140 patients, aged 20 to 70 years, divided into three groups: the 1st group had 74 patients with MDR-TB; the 2nd group consisted of 66 patients who had pulmonary TB without MDR; and the 3rd group consisted of 30 healthy donors. The 1st group was further divided into two subgroups: $1 \mathrm{~A}$, which had 41 patients with RPTB with MDR-TB, 1B - had 33 patients with newly diagnosed pulmonary tuberculosis (NDPTB) with MDR-TB. The 2nd group which had patients without MDR-TB was also divided into subgroups: $2 \mathrm{~A}$, consisting of 15 patients with RPTB; 2Bwith 51 patients with NDPTB. All patients had infiltrating pulmonary $\mathrm{TB}$ and were receiving standard chemotherapy consisting of daily doses of: isoniazid $(0.3 \mathrm{~g})$; rifampicin ( $0.6 \mathrm{~g})$; pyrazinamide $(2.0 \mathrm{~g})$; ethambutol $(1.2 \mathrm{~g})$; and/or streptomycin $(1.0$ g). Patients were recruited at the Regional TB Hospital № 1 and Regional TB Dispensary № 1. both in Kharkiv; Regional TB Dispensary № 3 in Zmeev and Regional TB Dispensary № 4 in Izyum, both in Kharkiv region.

Select parameters of LPO and AOS activities were evaluated

*Corresponding author: Dmytro O Butov, Kharkiv National Medical University Kharkiv, Ukraine, E-mail: dddimad@yandex.ua

Received October 16, 2013; Accepted December 17, 2013; Published December 19, 2013

Citation: Butov DO, Kuzhko MM, Kuznetsova IM, Grinishina OO, Maksimenko OA, et al. (2013) Dynamics of Oxidant-antioxidant System in Patients with Multidrug-resistant Tuberculosis Receiving Anti-mycobacterial Therapy. J Pulm Respir Med 3: 161. doi:10.4172/2161-105X. 1000161

Copyright: ( 2013 Butov DO, et al. This is an open-access article distributed under the terms of the Creative Commons Attribution License, which permits unrestricted use, distribution, and reproduction in any medium, provided the original author and source are credited. 
Citation: Butov DO, Kuzhko MM, Kuznetsova IM, Grinishina OO, Maksimenko OA, et al. (2013) Dynamics of Oxidant-antioxidant System in Patients with Multidrug-resistant Tuberculosis Receiving Anti-mycobacterial Therapy. J Pulm Respir Med 3: 161. doi:10.4172/2161-105X. 1000161

Page 2 of 3

by spectrophotometry in serum samples collected between 8 and 9 am prior to treatment initiation and 2 months later. The following parameters of LPO were measured: the content of conjugated Trienoic Fatty Acids (TC) and Lipofuscin Pigments (LP) [21]. The activity of AOS was evaluated by measuring thiol SH-groups [22], Total Antioxidant Activity (TAA) [23], Glutathione Peroxidase (GP) and Glutathione Reductase (GR) [24].

Obtained values were analyzed by the Student t-test. The difference was considered statistically significant at $\mathrm{P}<0.05[25]$.

\section{Results}

The patients with pulmonary TB exhibited significant perturbances in LPO and AOS activities as compared to healthy controls. The parameters of pro-oxidant system in TB patients, as shown in Table 1, namely the TC and LP levels, were significantly higher than in healthy donors $(\mathrm{p}<0.05)$. The AOS levels, i.e., GP, GR, TAA and SH-groups, as indicated in Table 2, were significant lower than in healthy donors $(p<0.05)$. In general in patients with pulmonary tuberculosis GP and TAA baseline values were almost twice lower and the LP is twice higher than in healthy donors.

Two months after treatment initiation with standard chemotherapy a significant reversal in indices of LPO and AOS activities was observed $(p<0.05)$. The levels of TC in LPO system in group 2 and $2 B$ after two months became practically same as in healthy controls $(\mathrm{p}>0.05)$ confirming relative recovery of LPO. As for the AOS the recovery was seen in GR and $\mathrm{SH}$ - levels other parameters were significantly different $(p<0.05)$ from healthy donors, suggesting the failure to recover after two months. For example levels of $\mathrm{SH}$ - in group 2 and $2 \mathrm{~B}$ were significantly higher than in healthy controls.

The comparison of MDR-TB patients (group 1) with those without MDR-TB (group 2) suggests a significant difference $(\mathrm{p}<0.05)$ in terms of reduction of almost all indicators as resulting from 2 months of chemotherapy. A significant difference between groups 1 and 2 was seen between treatment and post-treatment times and the gap was significantly higher in group 1 than in group $2(\mathrm{p}<0.05)$. The AOS indices were significantly higher in group 2 than in group 1 , both prior to treatment and after two months $(\mathrm{p}<0.05)$.

The comparison of patients with relapsed and newly diagnosed pulmonary tuberculosis indicates a significant reduction $(p<0.05)$ in all parameters before the treatment and after two months. The indicators of lipid peroxidation were significantly higher in patients with relapsed $\mathrm{TB}$ (1A and $2 \mathrm{~A}$ subgroups) than in patients NDPTB (subgroups $1 \mathrm{~B}$ and $2 \mathrm{~B})$ at both time points $(\mathrm{p}<0.05)$. In regard to the AOS significantly lower indices were seen in patients with RTBL ( $1 \mathrm{~A}$ and $2 \mathrm{~A}$ subgroups) than in NDPTB (1B and $2 \mathrm{~B}$ subgroups) before and after treatment $(\mathrm{p}<0.05)$. In addition, in patients with RPTB MDR-TB (subgroup 1A) the values were significantly lower than in RPTB without MDR-TB (subgroup 2A). In patients with NDPTB MDR-TB (subgroup 1B) the

\begin{tabular}{|c|c|c|c|c|}
\hline \multirow{2}{*}{ Groups and subgroups (n) } & \multicolumn{2}{|c|}{ Trienoic conjugates, $\mathrm{mmol} / \mathrm{L}$} & \multicolumn{2}{|c|}{ Lipofuscin pigments, $\mu \mathrm{mol} / \mathrm{L}$} \\
\hline & Before Rx & After 2 months & Before Rx & After 2 months \\
\hline $1(n=74)$ & $12.93 \pm 0.25^{\# \odot}$ & $9.05 \pm 0.21^{\# \Theta^{*}}$ & $1.78 \pm 0.05^{\# \oplus}$ & $1.2 \pm 0.04^{\# \Theta^{*}}$ \\
\hline $1 A(n=41)$ & $13.66 \pm 0.32^{\odot @}$ & $9.59 \pm 0.25^{\oplus^{*}}$ & $1.92 \pm 0.07^{\oplus @}$ & $1.33 \pm 0.05^{\odot^{*}}$ \\
\hline $1 B(n=33)$ & $12.03 \pm 0.35^{\odot \notin \otimes}$ & $8.38 \pm 0.31^{\oplus^{*} \phi \circledast}$ & $1.59 \pm 0.07^{\circledast \notin \circledast}$ & $1.04 \pm 0.04^{\odot^{*} \phi \circledast}$ \\
\hline $2(n=66)$ & $10.68 \pm 0.24^{\odot}$ & $7.21 \pm 0.21^{\alpha^{*}}$ & $1.41 \pm 0.05^{\odot}$ & $0.97 \pm 0.03^{\odot^{*}}$ \\
\hline $2 A(n=15)$ & $12.08 \pm 0.53^{\odot \bullet}$ & $8.24 \pm 0.35^{\odot *}$ & $1.63 \pm 0.12^{\odot}$ & $1.1 \pm 0.07^{\odot^{*}}$ \\
\hline $2 B(n=51)$ & $10.26 \pm 0.24^{\oplus a}$ & $6.92 \pm 0.24^{\alpha^{* a}}$ & $1.34 \pm 0.05^{\oplus x}$ & $0.94 \pm 0.03^{\odot^{*}}$ \\
\hline $3(n=30)$ & $7.05 \pm 0.22$ & $0.85 \pm 0.02$ & & \\
\hline
\end{tabular}

\# - difference significant $(p<0.001)$ when comparing between 1 and 2 group, ( $)$ - difference was significantly $(p<0.05)$ when compared with group 3 , $\alpha$ - difference is not significant $(p>0.05)$ when compared with group $3,{ }^{*}$ - difference was significantly $(p<0.001)$ when compared to treatment and after 2 months between the group (subgroup), $@$ - significant difference $(p<0.05)$ when comparing between $1 A$ and $2 A$ subgroup, $\phi$ - significant difference $(p<0.01)$ when comparing between $1 B$ and $2 B$ subgroup,

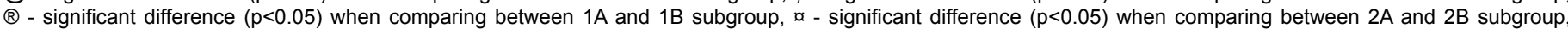
- - significant difference $(p<0.001)$ when comparing between $1 A$ and $2 B$ subgroup, $\bullet-$ the difference is not significant ( $p>0.05)$ when comparing between the $2 A$ and $1 B$ subgroup

Table 1: Parameters of lipid peroxidation in patients with pulmonary tuberculosis and healthy donors $(\mathrm{M} \pm \mathrm{m})$.

\begin{tabular}{|c|c|c|c|c|c|c|c|c|}
\hline \multirow{2}{*}{$\begin{array}{c}\text { Groups and } \\
\text { subgroups (n) }\end{array}$} & \multicolumn{2}{|c|}{ Glutathione peroxidase, s.u. } & \multicolumn{2}{|c|}{ Glutathione reductase, s.u. } & \multicolumn{2}{|c|}{ Total antioxidant activity, \% } & \multicolumn{2}{|c|}{ SH-groups, mmol/l } \\
\hline & to treatment & After two months & to treatment & after two months & to treatment & after two months & to treatment & after two months \\
\hline $1(n=74)$ & $3.416 \pm 0.09295^{\# \odot ~}$ & $4.562 \pm 0.1207^{\# O^{*}}$ & $134.7 \pm 1.911^{\# \odot}$ & $191 \pm 5.150^{\# \odot^{*}}$ & $20.72 \pm 0.5031^{\# \oplus}$ & $37.93 \pm 1.674^{\# 9^{*}}$ & $3.852 \pm 0.1389^{\# \oplus}$ & $5.969 \pm 0.1706^{\# 0^{*}}$ \\
\hline $1 A(n=41)$ & $3.139 \pm 0.09942^{\circ @}$ & $4.216 \pm 0.1218^{\odot^{*}} \mathrm{.}$ & $130.7 \pm 2.478^{\odot @-~}$ & $176 \pm 5.860^{\odot^{*} @}$ & $19.44 \pm 0.5037^{\odot} @$ & $33.95 \pm 1.327^{\odot * @-}$ & $3.259 \pm 0.1587^{\odot @-~}$ & $5.439 \pm 0.1888^{\odot^{*} @}$ \\
\hline $1 B(n=33)$ & $3.762 \pm 0.1488^{\oplus ф \circledast ~}$ & $4.993 \pm 0.2026^{\odot^{\star} \phi \circledast}$ & $139.6 \pm 2.785^{\oplus \notin \circledast}$ & $209.7 \pm 7.911^{\alpha^{*} \phi \circledast ~}$ & $22.30 \pm 0.8709^{\oplus \notin \circledast}$ & $42.88 \pm 3.197^{\odot^{\star} \phi \circledast}$ & $4.588 \pm 0.1703^{\odot \phi \circledast ~}$ & $6.627 \pm 0.263^{\alpha^{*} \oplus \otimes}$ \\
\hline $2(n=66)$ & $4.128 \pm 0.1058^{\odot}$ & $5.390 \pm 0.1280^{\odot^{*}}$ & $148.6 \pm 3.310^{\odot}$ & $228.7 \pm 5.461^{\alpha^{*}}$ & $24.65 \pm 0.6289^{\circ}$ & 51.44 & $4.883 \pm 0.1549^{\odot}$ & $0.2162^{\odot^{*}}$ \\
\hline $2 A(n=15)$ & $3.676 \pm 0.1637^{\odot}$ & $4.726 \pm 0.1624^{\odot^{*}}$ & $140.6 \pm 3.604^{\odot}$ & $206.3 \pm 9.687^{a^{*}}$ & $22.20 \pm 1.092^{\odot \bullet}$ & $39.53 \pm 1.502^{\odot^{*}}$ & $4.134 \pm 0.2883^{\odot}$ & $7.073 \pm 0.3317^{a^{*}}$ \\
\hline $2 B(n=51)$ & $4.261 \pm 0.1227^{\odot \mathrm{a}}$ & $5.585 \pm 0.1485^{\odot^{* a}}$ & $150.9 \pm 4.109^{\oplus \dagger}$ & $235.2 \pm 6.217^{\alpha^{*}}$ & $25.37 \pm 0.7222^{\oplus a}$ & $54.94 \pm 2.102^{\odot^{* a}}$ & $5.103 \pm 0.1711^{\oplus a}$ & $8.259 \pm 0.2513^{\Theta^{* \alpha}}$ \\
\hline $3(n=30)$ & $6.297 \pm 0.1985$ & & $217.6 \pm 5.901$ & & $64.73 \pm 1.1510$ & & $6.66 \pm 0.2588$ & \\
\hline
\end{tabular}

\# - difference significant $(p<0.01)$ when comparing between 1 and 2 group, $\odot$ - difference was significantly $(p<0.05)$ when compared with group 3 , $\alpha$ - difference is not significant $(p>0.05)$ when compared with group $3,{ }^{*}$ - difference was significantly $(p<0.001)$ when compared to treatment and after 2 months between the group (subgroup), $@$ - significant difference $(p<0.05)$ when comparing between $1 A$ and $2 A$ subgroup, $\phi$ significant difference $(p<0.01)$ when Comparing between $1 B$ and $2 B$ subgroup, $B$ - significant difference $(p<0.05)$ when comparing between $1 A$ and $1 B$ subgroup, $a$ - significant difference $(p<0.05)$ when comparing between $2 A$ and $2 B$ subgroup, $†-$ difference is not significant ( $p>0.05$ ) when comparing between $1 A$ and $2 B$ subgroup, $\mathbf{-}$ - significant difference $(p<0.001)$ when comparing between $1 A$ and $2 B$ subgroup, - - the difference is not significant $(p>0.05)$ when comparing between the $2 A$ and $1 B$ subgroup

Table 2: Condition indicators antioxidant defense in patients with pulmonary tuberculosis and healthy donors $(\mathrm{M} \pm \mathrm{m})$. 
Citation: Butov DO, Kuzhko MM, Kuznetsova IM, Grinishina OO, Maksimenko OA, et al. (2013) Dynamics of Oxidant-antioxidant System in Patients with Multidrug-resistant Tuberculosis Receiving Anti-mycobacterial Therapy. J Pulm Respir Med 3: 161. doi:10.4172/2161-105X. 1000161

Page 3 of 3

values were lower than in subgroup $2 \mathrm{~B}$ patients, and lipid peroxidation indices in patients with NDPTB without MDR-TB were also lower than in patients $1 \mathrm{~B}$ subgroup $(\mathrm{p}<0.05)$. The indicators of lipid peroxidation in subgroup $1 \mathrm{~A}$ were significantly higher than in subgroup $2 \mathrm{~A}(\mathrm{p}<0.05)$ at both time points.

\section{Conclusion}

We have conducted the measurements of antioxidant enzyme activity and lipid peroxidation parameters in $140 \mathrm{~TB}$ patients prior to and after two months on standard TB chemotherapy. In patients with pulmonary tuberculosis marked changes in the prooxidant-antioxidant system were observed as manifested by accumulation in the blood of LPO markers (TC and LP) and decreased function of the antioxidant system. Our data shows more pronounced disturbance in LPO and AOS in patients with relapsed and MDR-TB as compared to those who had newly diagnosed TB or were without MDR-TB. At the end of two months of standard anti-tuberculosis therapy we have observed relative normalization of parameters of LPO and AOS.

Increased activity of lipid peroxidation and decreased performance of AOS in patients with pulmonary tuberculosis provides a rationale to use in addition to standard TB therapy the antioxidant and membrane stabilizing interventions, especially in patients with relapsed MDR-TB.

\section{Acknowledgements}

We thank all volunteers who participated in this study. The wholehearted support of clinicians, nurses and lab personnel who contributed their effor made this study possible. Our gratitude is expressed to many experts in TB and immunology fields who kindly shared with us their opinions and critiques prior to and after this study was completed

\section{References}

1. World Health Organization (2011) Global tuberculosis control: report 2011 Geneva, Switzerland WHO/HTM/TB/2011.16.

2. World Health Organization (2008) The global burden of disease: 2004 update. Geneva, Switzerland WHO Document. W 74

3. Gandhi NR, Nunn P, Dheda K, Schaaf HS, Zignol M, et al. (2010) Multidrugresistant and extensively drug-resistant tuberculosis: a threat to global control of tuberculosis. Lancet 375: 1830-1843.

4. WHO (2012) Global Tuberculosis Report 2012.

5. Melnyk VM, Novozhylova IO, Matusevych VG, Linnyk MI (2012) Analytical view on a problem of drug-resistant tuberculosis: current status, achievements and unsolved issues. Ukrainian pulmonol $\mathrm{J}$ 1: 5-7.

6. Sokolova G, Bogadelnikova I, Byron M (2006) Clinical and radiological and bacteriological characteristics of drug-resistant tuberculosis. Problems of Tuberculosis and Lung Disease 12: 16-20.

7. Caminero JA (2010) Multidrug-resistant tuberculosis: epidemiology, risk factors and case finding. Int J Tuberc Lung Dis 14: 382-390.

8. Plieva SL, Seltsovskiy PP (2011) Features of early and late relapse of pulmonary tuberculosis. Tuberculosis and Lung Disease 6: 23-27.
9. Mishin VY, Zhestovskaya SN (2004) Relapses of pulmonary tuberculosis. Problems of Tuberculosis 4: 11-13.

10. Petrenko VM, Cherenko SO, Byalyk IB (2009) Evolution and effectiveness of antimycobacterial therapy in patients with pulmonary tuberculosis relapse. Ukrainian Pulmonol J 3: 14-19.

11. Volchegorsky IA, Ignatov GL, Volkova EG (1998) The relationship between the functional status of neutrophils and the intensity of peroxidation in the lungs in chronic bronchitis and ischemic heart disease. Bull Exp Biol 10: 452-454.

12. Volchegorsky IA, Popov AN (1993) Preoperative parameters of immune status and lipid peroxidation, a marker of the risk of pyelonephritis attack after percutaneous nefrolitektomii. Bull Exp Biol 8: 220-222

13. Savula MM, Kravchenko NS, Poznan Al (1993) Chemotherapy destructive pulmonary tuberculosis with use of antioxidants and antihypoxants. Problems of Tuberculosis 5: 18-20.

14. Safarian MD, Karapetian ET (1990) [Dynamics of the activity of antioxidan enzymes in the blood of patients with pulmonary tuberculosis]. Probl Tuberk: 60-61.

15. Lamsal M, Gautam N, Bhatta N, Toora BD, Bhattacharya SK, et al. (2007) Evaluation of lipid peroxidation product, nitrite and antioxidant levels in newly diagnosed and two months follow-up patients with pulmonary tuberculosis. Southeast Asian J Trop Med Public Health 38: 695-703.

16. Chornovil AV (2000) Lipid peroxidation and its pathogenetic correction in infectious diseases (review). Lviv Medical Journal 6: 17-22.

17. Dalvi SM, Patil VW, Ramraje NN, Phadtare JM, Gujarathi SU (2013) Nitric oxide, carbonyl protein, lipid peroxidation and correlation between antioxidant vitamins in different categories of pulmonary and extra pulmonary tuberculosis. Malays J Med Sci 20: 21-30.

18. Palanisamy GS, Kirk NM, Ackart DF, Obregón-Henao A, Shanley CA, et al. (2012) Uptake and accumulation of oxidized low-density lipoprotein during Mycobacterium tuberculosis infection in guinea pigs. PLoS One 7: e34148.

19. Palanisamy GS, Kirk NM, Ackart DF, Shanley CA, Orme IM, et al. (2011) Evidence for oxidative stress and defective antioxidant response in guinea pigs with tuberculosis. PLoS One 6: e26254.

20. Golubović S, Stanković I, Ristić L, Cosić V, Dordević I, et al. (2010) [Antioxidant enzymes and lipid peroxidation products in patients with pulmonary tuberculosis]. Med Pregl 63: 450-453.

21. Karpishchenko Al (1997) Medical Laboratory Diagnostics (programs and algorithms). St. Petersburg: "Intermedika".

22. Severin SE, Solovevoj TA (1989) Determination of the number of sulfhydryl groups with Ellman's reagent, Practicum in Biochemistry. Moscow: Moscow State University.

23. Bulankina NI, Ohrimenko SN, Ganusova GV (2005) Determination of total antioxidant capacity of blood plasma/Methods of lipids and carbohydrates: Methodical Instructions Special practical biochemistry. Kharkov.

24. Matushin BN, Loginov AS, Tkachev VD (1998) The activity of enzymes glutathione in chronic liver disease. Clinical lab diagnostics 4: 16-18.

25. Lapach SN, Chubenko AV, Babich PN (2000) Statistical methods in biomedical studies using Excel Kiev. 